PROCEEDINGS OF THE

AMERICAN MATHEMATICAL SOCIETY

Volume 134, Number 11, November 2006, Pages 3295-3299

S 0002-9939(06)08351-1

Article electronically published on May 8, 2006

\title{
AN INVERSE PROBLEM OF HAMILTONIAN DYNAMICS
}

\author{
M. RUDNEV AND V. TEN
}

(Communicated by Carmen C. Chicone)

\begin{abstract}
We study the question of whether for a natural Hamiltonian system on a two-dimensional compact configuration manifold, a single trajectory of sufficiently high energy is almost surely enough to reconstruct a real analytic potential.
\end{abstract}

Consider a compact configuration manifold $M^{n}$ equipped with a finite Borel measure (essentially we deal with the dimension $n=2$ ) and a natural Hamiltonian system thereon, with the Hamiltonian

$$
H(p, q)=\langle p, p\rangle_{q}+U(q), \quad(p, q) \in T^{*} M^{n} .
$$

Above, $\langle\cdot, \cdot\rangle_{q}$ is a Riemannian metric on $M^{n}$ and $U$ a potential. The direct problem of dynamics on $M^{n}$ is finding the trajectory $q(t) \subset M^{n}$, with initial conditions $q(0)=q_{0}$ and $\dot{q}(0)=v_{0}$, moving in the known force field $f(q)=-\nabla_{q} U(q)$ on $M^{n}$, where the gradient $\nabla_{q}$ has been associated with the metric $\langle\cdot, \cdot\rangle_{q}$.

Let us call the inverse problem of dynamics the problem of reconstruction of the potential by observing the system's trajectories $q(t)$. The first problem of this type was explored in Newton's Principia, in a quest for a physical law determining the planetary motion compatible with observational data 1 In the general case, knowledge of infinitely many trajectories is required to completely solve the problem. In this note we show that in the special case when $M^{n}$ is two dimensional, compact and topologically non-trivial, a single trajectory with sufficiently large energy would almost surely suffice to reconstruct the potential.

In the sequel, we assume that $M^{n}$ as well as all the quantities involved are realanalytic. Also suppose, there is an a-priori estimate $|U(q)|<C_{0}, \forall q \in M^{n}$, and we consider only the trajectories $q(t)$ with total energy $E \geq C_{0}$.

Theorem. Let $n=2$ and suppose $M^{2}$ is not diffeomorphic to $S^{2}$ or $\mathbb{R} P^{2}$. Almost every trajectory $q(t), t \geq 0$, with energy $E \geq C_{0}$, suffices to reconstruct the potential $U$ as a real-analytic function on $M^{2}$.

Let us recall the definition of a key set, or set of uniqueness; see e.g. 3 .

Definition. Let $D$ be a domain in $\mathbb{R}^{n}$ and $C^{\omega}(D)$ the class of real-analytic functions in $D$. A set $K \subset D$ is a key set if any $f \in C^{\omega}(D)$ vanishing identically on $K$ vanishes identically on $D$.

Received by the editors February 14, 2005 and, in revised form, May 26, 2005.

2000 Mathematics Subject Classification. Primary 37J05, 70H12.

${ }^{1}$ Note that the term "inverse problem of mechanics" has also been used to address the problem of deciding whether a given system of second order ODEs on $M^{n}$ has a Lagrangian; see e.g. [5].

(C)2006 American Mathematical Society Reverts to public domain 28 years from publication 
The theorem will follow from the following Lemma.

Lemma. If a real-analytic dynamical system $A: \dot{x}=F(x)$ on a compact phase space $P$ is non-singular (i.e. for no $x \in P, F(x)=0$ ) and the set of its closed orbits has positive measure, then all its orbits are closed.

Proof of the Lemma. Since the manifold $P$ is compact and the vector field $F$ is non-singular, there exists a Riemannian metric $g$ on $P$, such that in this metric the vector field $F$ has unit length at every $x \in P$. Furthermore, by compactness of $P$, the curvature (associated with $g$ ) of integral trajectories of $A$ is bounded from above, and therefore there exists some $T_{m}>0$, such that any periodic orbit of $A$ has period not smaller than $T_{m}$. This represents a particular case of the general result of Yorke ([7]).

Let us partition the range $\left[T_{m}, \infty\right)$ of possible periods (henceforth periods stand for minimum periods) for closed orbits into intervals of some small length $\delta_{1}$ to be specified. Let $I_{k}=\left[T_{m}+k \delta_{1}, T_{m}+(k+1) \delta_{1}\right) \equiv\left[T_{k}, T_{k+1}\right)$, for $k=0,1, \ldots$ Let $\Gamma_{k}$ be the set of all closed orbits, whose periods lie in $I_{k}$. Then for some $k=k_{*}$ the set $\Gamma_{*}=\Gamma_{k_{*}}$, considered as a subset of $P$, has positive measure. (We refer to the sets $\Gamma$ either as point sets or sets of orbits, depending on the context. As there are only measure-theoretical considerations involved, this should not cause confusion.)

Let $D_{\delta_{2}}(x)$ be a codimension one disk in $P$, centered at some $x \in P$, with radius $\delta_{2}$ and perpendicular (in the sense of metric $g$ ) to the vector field $F(x)$ at the point $x$. Let $\delta_{2}$ be small enough, so that the vector field is transversal to $D_{\delta_{2}}(x)$ at every point of the disk. Clearly, $\delta_{2}$ can be taken as a universal constant independent of $x$.

Let $x_{0}$ be a Lebesgue point of the set $\Gamma_{*}$. Recall that at a Lebesgue point, the density of the set is one. Let $\gamma_{0}$ be the closed trajectory passing through $x_{0}$, so $\gamma_{0} \in \Gamma_{*}$. Take a disk $D_{\delta_{2}}\left(x_{0}\right)$.

Then there is a well-defined analytic Poincaré map $S$ from a disk $D_{\epsilon}\left(x_{0}\right)$ contained in $D_{\delta_{2}}\left(x_{0}\right)$, where $\epsilon<\frac{1}{C_{1} T_{k_{*}}} \delta_{2}$, for some $C_{1}=C_{1}\left(\delta_{1}\right)$, and $x_{0}$ is a fixed point of $S$. On the disk $D_{\epsilon}\left(x_{0}\right)$, points that are initial conditions for orbits from $\Gamma_{*}$ form a set of positive measure, as $x_{0}$ is a Lebesgue point. Besides, the quantity $\delta_{1}<T_{m}$ can be chosen small enough to ensure that any $x \in D_{\epsilon}\left(x_{0}\right) \cap \Gamma_{*}$ is also a fixed point of the map $S$.

The union of all $x \in D_{\epsilon}\left(x_{0}\right) \cap \Gamma_{*}$ is a positive measure subset of $D_{\epsilon}\left(x_{0}\right)$, and hence is a key set (see [6] for the proof that every set of positive measure is a key set). Therefore, every point of $D_{\epsilon}\left(x_{0}\right)$ is an equilibrium of the map $S$, and hence an initial condition for a periodic orbit of $A$. Let $\Gamma_{\epsilon}$ be the union of all such orbits, with initial conditions in $D_{\epsilon}\left(x_{0}\right)$. Let $\Gamma \subseteq P$ be the maximum connected open set, which contains $\Gamma_{\epsilon}$ and is a union of periodic orbits.

To complete the proof, let us show that the set $\Gamma$ does not have a boundary, i.e. $\Gamma=P$. To show it, we use the following Gronwall-type estimate.

Proposition. Let $\phi(s) \subset \Gamma$ be a curve of length $L$, where $s$ is a natural parameter with respect to the metric $g$. Then for all $s \in[0, L]$ and some absolute constant $C_{2}$,

$$
T(\phi(s)) \leq T(\phi(0)) e^{C_{2} L}
$$

where $T(\phi(s))$ is the period of the closed orbit passing through the point $\phi(s)$. 
Indeed, the Proposition follows immediately from the following infinitesimal estimate: for some $C_{2}$,

$$
\left|\frac{d}{d s} T(\phi(s))\right| \leq C_{2} T(\phi(s)) .
$$

Returning to the proof of the lemma, suppose the boundary $\partial \Gamma$ is non-empty. As $\partial \Gamma$ is a compact set, the distance (in the sense of metric $g$ ) between $\partial \Gamma$ and the above-mentioned point $x_{0}$ attains its minimum at some point $y \in \partial \Gamma$. Connect $x_{0}$ and $y$ by a geodesic segment. Let the latter segment have length $L$; clearly all its points, except $y$, belong to $\Gamma$. Let $\gamma_{y}$ be the trajectory of $A$ with initial condition $y$.

For any point $x_{1} \neq y$ on the above geodesic segment, there is a uniform bound for the period of the corresponding closed orbit, by the Proposition. Hence, for any such $x_{1}$, there exists a uniform $\varepsilon$ (one can take $\varepsilon=\epsilon e^{-C_{2} L}$, where $\epsilon$ has been defined earlier) such that an analytic Poincaré map can be defined in exactly the same way as $S$ above, but now with the domain $D_{\varepsilon}\left(x_{1}\right)$. Choosing $x_{1}$ such that the intersection $\gamma_{y} \cap D_{\varepsilon}\left(x_{1}\right)$ is not empty and repeating the key set argument leads to contradiction: all orbits in some tubular neighborhood of $\gamma_{y}$, including $\gamma_{y}$ itself, are periodic.

Proof of the Theorem. Consider a randomly chosen trajectory $\gamma$ on some energy level $H^{-1}(E), E \geq C_{0}$, which is obviously a non-critical level.

According to the Lemma, either (i) all the trajectories on $H^{-1}(E)$ are periodic, or (ii) a randomly chosen initial condition $\left(p_{0}, q_{0}\right) \in H^{-1}(E)$ results in a phase trajectory of infinite length almost surely.

The former case (i) may occur only if $M^{2}$ is a so-called $P$-manifold. Indeed, according to the Maupertuis principle, the phase trajectories of motions with total energy $E$ project onto $M^{2}$ as geodesic lines of the corresponding (Riemannian) Jacobi metric. $P$-manifolds are Riemannian manifolds, all of whose geodesics are closed; see [1. Topological properties of $P$-manifolds are characterized in great detail in various dimensions within the framework of the Bott-Samelson theorem. In our (simplest possible) case, it is easy to see that $M^{2}$ can only be diffeomorphic to either $S^{2}$ or $\mathbb{R} P^{2}$.

Indeed, the proof of the lemma implies that all the (closed) phase trajectories on the energy level $E$ are homotopic to one another. Then their images on $M^{2}$ (under natural projection) are also homotopic. On the other hand, if $M^{2}$ is different from $S^{2}$ or $\mathbb{R} P^{2}$, the number of generators for its fundamental group equals at least two. As for any Riemannian metric there exist closed geodesics in each free homotopy class, we would have a contradiction, unless $M^{2}$ is $S^{2}$ or $\mathbb{R} P^{2}$.

In the case (ii), let $q(t)=\left(q_{1}(t), q_{2}(t)\right)$ be a randomly chosen trajectory: it almost surely has infinite length. Clearly, we can easily derive the gradient of $U$ at every point of the trajectory from the Euler-Lagrange equations.

Thus to complete the proof of the Theorem, let us show that any non-closed trajectory is a key set. Consider orbit segments $\{q(t), t \in[k, k+1)\}, k=0,1, \ldots$, in $M^{2}$. (Note that time can always be scaled to ensure that each segment is a simple curve in $M^{2}$, or shorter time intervals can be considered.) As $M^{2}$ is compact, there is a limit point $q_{*}$ of the point sequence $\{q(k+1 / 2)\}$. Consider a sufficiently small circle centered at $q_{*}$. There are two options. Either the circle intersects the trajectory $q(t)$ at infinitely many points, or at some point on the circle the trajectory $q(t)$ intersects itself (transversely as it is a geodesic) infinitely many times. In the 
latter case, take the point of infinite self-intersection for $q_{*}$, otherwise leave $q_{*}$ as it is. In either case, there exists a point $q_{*}$, with the property that any sufficiently small circle centered at $q_{*}$ is intersected by the trajectory $q(t)$ infinitely many times.

Therefore, the force $f$ and the potential $U$ can be uniquely reconstructed on any sufficiently small circle centered at $q_{*}$ (an infinite point set on a circle is a key set for the circle), and therefore in some neighborhood of $q_{*}$, and hence on the whole configuration manifold $M^{2}$.

In conclusion, let us make several remarks.

1. The Theorem can be stated in terms of analytic geodesic flows on compact Riemannian 2-manifolds. Namely, if $M^{2}$ is not diffeomorphic to a sphere or real projective plane, a randomly chosen geodesic suffices to reconstruct the metric, almost surely. Indeed, any Riemannian metric is locally conformal to the Euclidean one, i.e. can be locally associated with the Hamiltonian $H(p, q)=e^{\rho\left(q_{1}, q_{2}\right)}\left(p_{1}^{2}+p_{2}^{2}\right)$. Our Theorem enables one to reconstruct the real-analytic function $\rho\left(q_{1}, q_{2}\right)$ locally near $q_{*}$ from the Hamilton equations, with subsequent analytic continuation to get the metric globally on $M^{2}$.

2. The Theorem is essentially two dimensional, as for $n \geq 3$ the fact that a random trajectory has infinite length does not suffice to reconstruct the potential. Consider for instance the Euler top, where $M^{n}=S O(3)$. In this case, the phase space is foliated by invariant two-tori, where the trajectories are in general conditionally periodic. Clearly, a projection of a single invariant two-torus onto the three-dimensional configuration space is not a key set.

3. The lemma does not apply to the case of invariant tori of dimension higher than one. Indeed, a particular case of the KAM theorem states that a sufficiently small perturbation of a non-degenerate Liouville-integrable Hamiltonian system in $T^{*} M^{n}$ yields a positive measure set of invariant $n$-tori that do not fill the whole energy surface.

4. In the special case $M^{2}=\mathbb{R} P^{2}$, by the theorem of L. Green (4]), the only metric for which all geodesics are closed is the standard metric. The case $M^{2}=S^{2}$ has been a subject of extensive research for over 100 years, arguably beginning with the doctoral thesis of $\mathrm{O}$. Zoll ( 8$]$ ). The reader is referred to the excellent book by L. Besse ([1]), which gives the issue a thorough treatment.

5. The condition $E \geq C_{0}$ seems unavoidable. For small energies the domain of possible motions can be a disk, with the Jacobi metric degenerate on the boundary, in which case one may expect a scenario similar to the case $M^{2}=S^{2}$.

6. Observe that in the exceptional case when $M^{2}$ is a $P$-manifold, the geodesic flow thereon is completely integrable (see e.g. 2] for the proof of this fact). Hence our theorem implies that if $n=2$, it is sufficient for restoration of the potential almost surely from a single trajectory that the system possess no other analytic integrals of motion but energy. It seems likely that in the latter weaker formulation the theorem should extend to the case $n>2$, however we do not know how to prove it.

\section{REFERENCES}

[1] A. Besse. Manifolds all of whose Geodesics are Closed. Springer-Verlag, Berlin, Heidelberg, New York, 1978. MR0496885 (80c:53044)

[2] A.V. Bolsinov, B. Jovanović. Noncommutative integrability, moment map and geodesic flows. Ann. Global Anal. Geom. 23 (2003), no. 4, 305-322. MR1972543 (2004b:37113) 
[3] V.I. Arnold, V.V. Kozlov, A.I. Neishtadt. Encyclopedia of Mathematical Sciences. Dynamical Systems III. Mathematical Aspects of Classical and Celestial Mechanics. Springer-Verlag, Berlin, 1988. MR1292466 (95d:58043b)

[4] L.W. Green. Auf Wiedersehensflächen. Ann. of Math. 78 (1963), no. 2, 289-299. MR0155271 $(27: 5206)$

[5] M. de León, P.R. Rodrigues. Methods of differential geometry in analytical mechanics. North-Holland Mathematics Studies 158. North-Holland Publishing Co., Amsterdam, 1989. MR1021489 (91c:58041)

[6] V.V. Ten. Analytic invariants of dynamical systems with positive entropy. (Russian). Vestnik Moskov. Univ. Ser. I Mat. Mekh. (1997), no. 3, 40-43. MR1483040(98g:58107)

[7] J.A. Yorke. Periods of periodic solutions and the Lipschitz constant. Proc. Amer. Math. Soc. 22 (1969), 509-512. MR0245916 (39:7222)

[8] O. Zoll. Über Flächen mit Scharen geschlossener geodätischer Linien. (German). Math. Ann. 57 (1903), 108-133. MR1511201

Department of Mathematics, University of Bristol, University Walk, Bristol BS8 1TW, United Kingdom

E-mail address: m.rudnev@bris.ac.uk

Department of Mathematics, University of Bristol, University Walk, Bristol BS8 1TW, United Kingdom

E-mail address: v.ten@bris.ac.uk 